\title{
Place specificity of monopolar and tripolar stimuli in cochlear implants: The influence of residual masking ${ }^{\text {a) }}$
}

\author{
Claire A. Fielden, ${ }^{\text {b) }}$ Karolina Kluk, and Colette M. McKay \\ School of Psychological Sciences, Ellen Wilkinson Building, University of Manchester, Oxford Road, \\ Manchester M13 9PL, United Kingdom
}

(Received 21 June 2012; revised 25 March 2013; accepted 19 April 2013)

\begin{abstract}
This experiment investigated whether place specificity of neural activity evoked by cochlear implant stimulation is improved in tripolar compared to monopolar mode using a forward masking protocol addressing some limitations of previous methods of measurement and analysis. The amount of residual masking (masking remaining at long masker-probe delays) was also measured, and its potential influence on the specificity measures was evaluated. The masker stimulus comprised equally loud interleaved mono- or tripolar stimulation on two electrodes equidistant from a central probe electrode in an apical and basal direction, reducing the influence of off-site listening. The effect of masker-probe distance on the threshold shift of the tripolar probe was analyzed to derive a measure of place specificity. On average, tripolar maskers were more place specific than monopolar maskers, although the mean effect was small. There was no significant effect of masker level on specificity or on the differences observed between modes. The mean influence of residual masking on normalized masking functions was similar for the two modes and, therefore, did not influence the comparison of specificity between the modes. However, variability in amount of residual masking was observed between subjects, and therefore should be considered in forward masking studies that compare place specificity across subjects. (C) 2013 Acoustical Society of America. [http://dx.doi.org/10.1121/1.4803909]
\end{abstract}

PACS number(s): 43.64.Me, 43.66.Dc [KG]

Pages: 4109-4123

\section{INTRODUCTION}

Compared with 3000 tonotopically organized inner hair cells in a normally hearing cochlea, cochlear implants are restricted both by small numbers of channels and by non-specific neural excitation. In a multichannel cochlear implant, electrode activation results in a wide electrical field and inevitably a degree of overlap in neural excitation between channels. Channel interactions have been demonstrated, both psychoacoustically (de Balthasar et al., 2003; Kwon and van den Honert, 2006; Stickney et al., 2006) and physiologically (Bierer and Middlebrooks, 2002; Middlebrooks, 2004). Tripolar stimulation has been proposed as a method to focus the electrical field and thus produce more place-specific neural activation in the cochlea. This study compared the place specificity of monopolar (MP) and tripolar (TP) stimulation using a new forward masking paradigm designed to overcome several of the methodological limitations of previous studies. The method limited off-frequency (or off-site) listening as well as other potentially confounding effects such as loudness or dynamic range differences between modes and electrodes. In addition, the possible influence of residual masking (probe threshold

\footnotetext{
${ }^{\text {a) }}$ Portions of this work were presented in a poster at the Conference for Implantable Prostheses, Asilomar, CA, July 2011.

${ }^{b)}$ Author to whom correspondence should be addressed. Also at: Midlands Hearing Implant Programme, Queen Elizabeth Hospital Audiology Centre, Nuffield House, Mindelsohn Way, Edgbaston, Birmingham, B15 2TH, UK. Electronic mail: claire.fielden@postgrad.manchester.ac.uk.
}

shift that persists after a long masker-probe delay) on place specificity measures was evaluated.

Studies investigating temporal recovery from forward masking, for example, Dingemanse et al. (2006) and Nelson and Donaldson (2002), have demonstrated that the probe threshold shift generally does not decay to zero for long masker-probe temporal offsets, but asymptotes to a residual constant, termed "residual masking," or incomplete recovery from forward masking. The mechanism responsible for residual masking, also observed in acoustic forward masking experiments (Oxenham and Moore, 1995), is not yet fully understood but is likely related to cognitive factors associated with the listening task itself (McKay, 2012) and therefore may also contribute to the threshold shift measured at the shorter masker-probe delays used to derive spatial forward masking functions (the effect of masker-probe distance on forward masking). Previous studies that determined spatial forward masking functions have not considered the possible influence of residual masking on the shape of the spatial masking function and hence on the measure of specificity derived from this function. If residual masking is a cognitive effect that varies between individuals, it may affect the specificity measure differently in different people.

Typically, single-electrode maskers have been used in forward masking studies that investigated place specificity (Lim et al., 1989; Cohen et al., 1996; Chatterjee and Shannon, 1998; Throckmorton and Collins, 1999; Cohen et al., 2003; Chatterjee et al., 2006; Kwon and van den Honert, 2006; Hughes and Stille, 2008; Nelson et al., 2008; Bierer and Faulkner, 2010; Macherey et al., 2010). However, methods using a single-electrode masker do not control for off-site 
listening, i.e., attending to neural activity due to the probe at a distal location from the probe electrode on the side opposite the masker. Off-site listening has the effect of artificially sharpening the spatial forward masking function and thus overestimating the specificity (Moore and Glasberg, 1981). In an analogous approach to notched-noise masking in acoustic hearing (Seaton, 1979), Dingemanse et al. (2006) used a dualelectrode masker, with one masker electrode on either side of and equidistant from, the probe electrode to limit off-site listening. They found a consistently wider spatial forwardmasking function when using a dual-electrode compared to a single-electrode masker, demonstrating the effect of off-site listening on specificity measures. Dual-electrode maskers were used to limit off-site listening in the current experiment. In contrast to Dingemanse et al., who used maskers of equal current, the masker current levels in this experiment were adjusted to evoke equal loudness across masker electrodes, thus taking into consideration potential differences in thresholds or dynamic ranges across masker positions.

Three previous papers (Bierer and Faulkner, 2010; Zhu et al., 2012; Landsberger et al., 2012) have compared place specificity of TP and MP stimulation using psychophysical forward masking and have reported variable and modest advantages of TP. These small effects are surprising given that physiological studies have shown a greater advantage for TP (Kral et al., 1998; Bierer and Middlebrooks, 2002; Bierer et al., 2010). It is notable that none of the psychophysical papers used a method that limited off-site listening, the potential influence of which is likely to differ between the modes. For example, if the probes differ in mode [as was the case for Bierer and Faulkner (2010) and Zhu et al. (2012)], the MP probe will provide more opportunity for off-site listening than a TP probe if the neural excitation due to the MP probe had the wider extent. This effect would sharpen the MP function more than the TP function, decreasing the apparent difference between the modes. One of the objectives of this paper was to use a forward masking method that limited off-site listening to investigate whether a more consistent difference between the modes could be seen compared to previous research.

In addition to off-site listening, each of the preceding papers (Bierer and Faulkner, 2010; Zhu et al., 2012; Landsberger et al., 2012) used measurement or analysis methods that did not ideally limit the effects of other possible factors that may confound the comparison of modes. McKay (2012) recently published a review of measurement and analysis methods that showed that specificity measures can be greatly influenced by the methods used to obtain them, and the reader is referred to this review for more detailed theoretical explanations of the issues discussed here. Landsberger et al. (2012) used a masker fixed in location and level (varying in tripolarity) and measured the masked and unmasked probe thresholds (in TP with $\sigma=0.75$ ) on different electrodes to obtain functions of probe threshold shift versus probe position. The threshold shift was calculated by subtracting masked and unmasked probe currents in $\mu \mathrm{A}$, and the measure of specificity was the area under the function of normalized threshold shift (threshold shift divided by the shift with probe on the masker electrode) versus probe electrode. Calculating threshold shift by subtracting currents (instead of calculating the ratio or $\mathrm{dB}$ change), however, can severely distort the masking function if the unmasked probe threshold varies across electrodes and can change the apparent specificity across subjects or modes depending on the absolute current levels used (McKay, 2012). Given that TP thresholds vary significantly across electrode positions (Bierer, 2007), the influence of the subtraction measure on the shape of the forward masking function could be considerable, for instance, producing functions with a shifted tip. In addition, the specificity measure used (area under the forward masking functions) is difficult to validate in cases where the function is truncated at the edges, as it was in all six subjects in this paper.

Bierer and Faulkner (2010) used the "spatial tuning curve" method in which the probe stimulus is fixed in level and position and the masker is varied in position and level to just mask the probe. The maskers were always in TP mode, and the probe mode was either MP or TP. The masking functions were thus masker level (plotted in both $\mathrm{dB}$ and percent dynamic range-\%DR) versus masker position. Two measures of specificity were used: the slope of the function in $\mathrm{dB} /$ $\mathrm{mm}$ and the width of the function plotted in $\% \mathrm{DR}$ at a point halfway between the tip and maximum masker level. The first measure is not an ideal measure of specificity as the slope of the masker level function depends both on specificity and the slope of the masker thresholds across the masker electrodes, the latter being likely to be flatter for MP compared to TP maskers leading to more variability in the TP measure. The second measure is not valid as it depends critically on probe level and highest masker used. More appropriate measures would have been the slope of the functions in $\% \mathrm{DR} / \mathrm{mm}$ or the width at a fixed level (in \%DR) above the tip (McKay, 2012). Additionally, the modes were compared for probes of a fixed sensation level in $\mathrm{dB}$, whereas equating the probes in loudness may have produced a more useful comparison if the probes had different dynamic ranges or loudness growth.

Zhu et al. (2012) also used the spatial tuning curve method, where the probe was fixed in position and level and the maskers on different electrodes were adjusted to just mask the probe, and plotted the masker level versus masker position in $\mathrm{dB}$ re $1 \mu \mathrm{A}$. In contrast to the previous two studies, masker and probe were the same mode (thus spatial tuning curves for the different modes represented maskers of different modes). This latter point makes it difficult to compare the widths across the different modes, and the authors did not say how they defined the width so it is unknown whether their measure was a valid one. As with the Bierer and Faulkner (2010) paper, the slopes of the functions using masker level in $\mathrm{dB}$ (and hence the width) may not be wholly dependent on the specificity alone. Although the probes were set at broadly the same \%DR in each mode, they were not loudness balanced, introducing a potential variability or bias in the results.

In summary, previous studies that compared place specificity of TP with MP mode using forward masking included factors or methods that may have led to unhelpful variability or confounding effects in the comparison. This may partly 
explain the variable and modest effect of mode found on the specificity measures. In particular, no study has limited offsite listening when comparing modes and the effect of residual masking on spatial forward masking functions has not been evaluated. The present study investigates the effect of residual masking using a dual-electrode masker to limit off-site listening, as well as carefully controlling the measurement and analysis methods to avoid confounding the comparison of modes.

\section{METHODS}

\section{A. Participants}

Nine post-lingually deafened adults fitted with a HiRes $90 \mathrm{~K}$ device (Advanced Bionics Corp., Sylmar, CA) participated in this study. All participants were successful implant users with some measurable open set sentence discrimination in quiet (see Table I for sentence scores at $70 \mathrm{dBA}$ in quiet). All participants had a full electrode insertion confirmed via $\mathrm{x}$-ray, with all 16 electrodes activated and no known electrode anomalies. All participants used the HiRes120 speech processing strategy for their everyday listening. All participants were unilateral implant users except S4 who wore simultaneously fitted bilateral implants. Only her right implant was used in the study. One participant (S9) was fitted with a HiFocus Helix electrode array, the remaining eight received a HiFocus $1 \mathrm{~J}$ electrode array. Participant demographics are summarized in Table I. The study received ethical approval from Northwest 7 Research Ethics Committee at Central Manchester Foundation Trust, UK. Ethical guidelines outlined by the Declaration of Helsinki were adhered to, and each participant provided written consent to participate in the study.

\section{B. Equipment and stimuli}

TP electrode configuration utilizes three intracochlear electrodes with the active electrode referenced to the two adjacent electrodes. Partial tripolar (pTP) mode utilizes the extracochlear electrode as a further reference electrode and the degree of current focusing can be varied by changing the amount of return current sent to this electrode (Fig. 1). The parameter $\sigma$ defines the proportion of current that is returned to the intracochlear electrodes with $\sigma=1$ representing a full TP mode and $\sigma=0$ equivalent to MP mode.
The HiRes $90 \mathrm{~K}$ uses 16 intracochlear electrodes, plus an extracochlear reference electrode situated on the receiver/ stimulator package. Electrodes are labeled 1-16 with electrode 1 being the most apical and electrode 16 the most basal. Center-center electrode distance is $1.1 \mathrm{~mm}$ in the HiFocus $1 \mathrm{~J}$ and $0.85 \mathrm{~mm}$ in the HiFocus Helix arrays. Multiple independent current sources allow easy manipulation of mode of stimulation. All stimuli were generated using the BIONIC EAR DATA COLLECTION SYSTEM version 1.17.210, and psychophysical responses were measured using the PSYCHOACOUSTICS TEST SUITE version 1.7 .7 , both provided by Advanced Bionics Corp.

Throughout the experiment, the TP mode was partial tripolar (pTP) with a focusing coefficient $(\sigma)$ of 0.75 (see Fig. 1), selected to avoid the nodes of current at the adjacent electrodes in full TP mode that may be perceivable by the implant user (Jolly et al., 1996). Wherever the term TP is used hereafter in the context of the present experiment, it refers to TP stimulation with this coefficient. In the forward masking paradigm, the probe stimulus was always TP mode to limit the effects of current spread from the probe, whereas the masker stimulus was either TP or MP modes, so any differences in probe threshold shift between masker modes could be attributed to the masker mode alone.

The probe stimulus was on electrode 7 , chosen due to its central location along the array. The two electrodes in the dual-electrode masker were located basally and apically at equal distances away from electrode 7 , for example, a masker-probe distance of 3 denotes a masker stimulus comprising alternating and sequential pulses on electrodes 4 and 10 (i.e., a distance of 3 electrodes away from electrode 7 in both an apical and basal direction). In the forward masking procedure, a 300-ms-duration masker pulse train preceded a 20 -ms-duration probe pulse train with a delay of $30 \mathrm{~ms}$ as measured by the time between the offset of the masker stimulus and the offset of the probe stimulus [see Fig. 2(A)].

In the dual-electrode masker stimulus, pulses on the masker electrodes were presented in an interleaved fashion with an overall rate of $4640 \mathrm{pps}$, identical to the rate of the probe stimulus [see Fig. 2(B) for diagrammatic representation of probe and masker pulse trains]. Pulse durations were $43.1 \mu \mathrm{s}$, and interphase gaps were $21.55 \mu \mathrm{s}$. The currents on the two masker electrodes were those that produced equally loud percepts (across electrodes and across modes). These

TABLE I. Participant demographics. BKB refers to the most recent Bamford-Kowal-Bench test of open-set sentence discrimination in quiet (Bench et al., 1979); DPD is the duration of profound deafness.

\begin{tabular}{|c|c|c|c|c|c|c|c|}
\hline ID & Age (years) & Gender & Electrode array & Etiology & Date of CI & DPD (years) & BKB score $(\%)$ \\
\hline $\mathrm{S} 1$ & 78 & $\mathrm{M}$ & HiFocus $1 \mathrm{~J}$ & Unknown & $10 / 02 / 2007$ & 2 & 69 \\
\hline $\mathrm{S} 2$ & 69 & $\mathrm{~F}$ & HiFocus $1 \mathrm{~J}$ & Meniere's disease & $16 / 10 / 2007$ & 7 & 98 \\
\hline S3 & 82 & $\mathrm{~F}$ & HiFocus $1 \mathrm{~J}$ & Hereditary & 03/06/2008 & 2 & 92 \\
\hline $\mathrm{S} 4$ & 55 & $\mathrm{~F}$ & HiFocus $1 \mathrm{~J}$ & Flynn Airds Syndrome & $24 / 05 / 2007$ & 10 & 83 \\
\hline S5 & 81 & $\mathrm{~F}$ & HiFocus $1 \mathrm{~J}$ & Viral infection & $05 / 08 / 2008$ & 5 & 65 \\
\hline S6 & 79 & M & HiFocus $1 \mathrm{~J}$ & Noise induced & $06 / 06 / 2008$ & 5 & 54 \\
\hline S7 & 67 & M & HiFocus $1 \mathrm{~J}$ & Measles & 03/10/2006 & 2 & 92 \\
\hline S9 & 46 & $\mathrm{~F}$ & HiFocus Helix & Multiple sclerosis & $01 / 05 / 2007$ & 8 & 95 \\
\hline S10 & 56 & $\mathrm{~F}$ & HiFocus $1 \mathrm{~J}$ & Hereditary & $17 / 01 / 2006$ & 23 & 75 \\
\hline
\end{tabular}




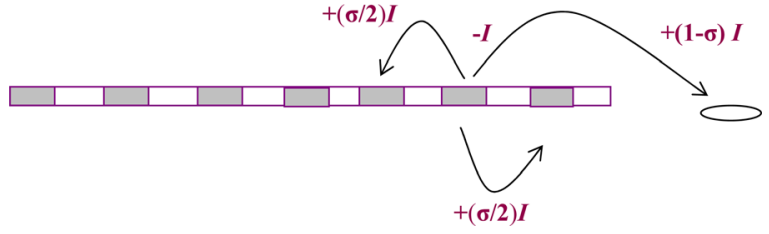

FIG. 1. (Color online) Diagrammatic representation of partial tripolarity (pTP), with the oval shape representing the extracochlear electrode. MP has a sigma (tripolarity) value of 0 , whereas full TP has a sigma value of 1 .

currents were determined using a loudness balancing task described in Sec. II C 2. Software used in this experiment controlled the current in $\mu \mathrm{A}$ units; however, all analyses were performed using a $\mathrm{dB}$ current scale, including the averaging of multiple measures such as thresholds and loudness balancing runs. Forward masking measurements were taken at two masker sensation levels, comfortably loud and comfortably soft, and at six masker-probe distances: 0, 1, 2, 3, 4, and 5 electrode spacings.

Residual masking (i.e., the probe threshold shift remaining at long temporal offsets) was measured using an increased masker-probe temporal offset of $220 \mathrm{~ms}$. This offset was chosen as implant listeners are likely to demonstrate time constants for recovery from forward masking of less than $95 \mathrm{~ms}$ (Nelson and Donaldson, 2002). To investigate whether masker level and masker-probe distance would influence the degree of residual masking, measurements were taken at two masker levels (comfortably loud and comfortably soft) and at two masker-probe distances (0 and 5).

\section{Procedure}

\section{Measuring threshold, maximum acceptable levels and most comfortable levels on masker electrodes}

First, the ranges of appropriate currents for masker electrodes contributing to the dual-electrode maskers were determined for the two modes. Using a loudness category scale with categories ranging from "cannot hear" to "too loud," values of threshold level, "comfortably soft," "comfortably loud," and maximum acceptable level (MAL) were determined with cannot hear corresponding to the level below threshold and too loud corresponding to the MAL. Stimuli were initially presented at very low levels of current, usually sub-threshold, and increased in steps of $5 \mu \mathrm{A}$ until the participant had indicated MAL. Threshold and MALs were obtained on the current ascent, but approximate comfortably loud and comfortably soft levels were obtained on the descent. Stimuli for this task and the following loudness balance task were single-electrode stimuli in either MP or TP mode, of $300 \mathrm{~ms}$ duration with the same overall rate as the eventual dual-electrode masker (4640 pps). The overall masker rate, rather than the singlecomponent masker rate (2320 pps), was used because it was predicted that when the loudness-balanced currents were used in a dual-electrode masker with the same overall rate, a similar loudness to the single-electrode stimuli would be perceived, and therefore the dual-electrode stimulus would not need further adjustment for comfort (McKay et al., 2003). In each case, no such adjustment was needed, as anticipated.

\section{Loudness balancing of masker electrodes}

The single-electrode stimulus in TP mode on electrode 7 was first adjusted to comfortably loud (hereafter referred to as "loud") as indicated by the participant. Single-electrode TP stimuli on all other masker electrodes were then balanced for loudness to this stimulus using an adaptive two-interval two-alternative forced choice (2I2AFC) procedure in which the participant was presented with pairs of stimuli: One on the reference electrode and one on the "test" electrode to be balanced. Participants were instructed to select the louder sound of the pair and to respond even if they were unsure which was louder. The test stimulus level was adjusted using a one-down one-up stepping rule that estimated the test level

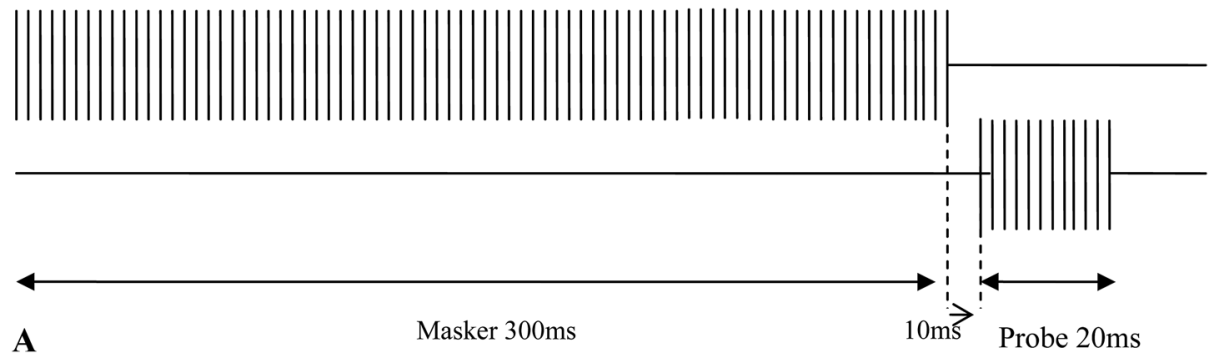

\footnotetext{
FIG. 2. (A) contains a schematic diagram of the masker/probe forward masking paradigm. The dual-electrode masker comprised a $300 \mathrm{~ms}$ pulse train of interleaved pulses on two variable "capturing" electrodes. The $20 \mathrm{~ms}$ probe stimulus on electrode 7 followed the masker stimulus after a gap of $10 \mathrm{~ms}$ ( $30 \mathrm{~ms}$ masker-probe offset). The probe stimulus was always in TP mode, whereas the masker stimulus was in either MP or TP modes. (B) demonstrates the temporal pattern of pulses on masker and probe electrodes. All pulses were presented cathodic first. Note that the overall rate of the two masker electrodes in the dual-electrode masker is equal to the rate of the probe stimulus.
}

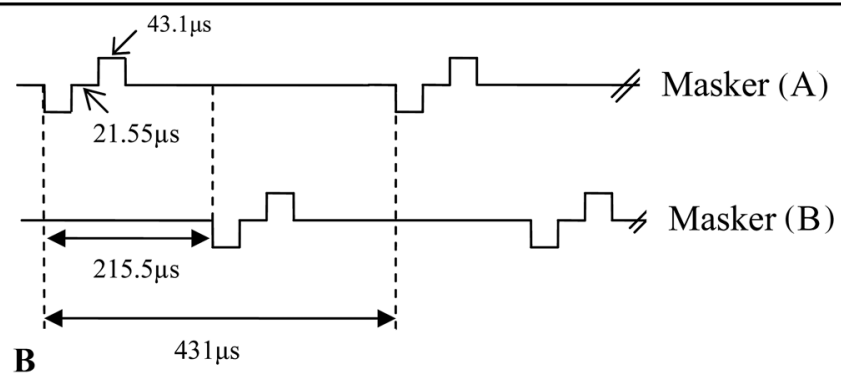


judged to produce equal loudness to the reference stimulus. Levels were adjusted by $20 \%$ (in $\mu \mathrm{A}$ ) until the first reversal and then by $10 \%$ until the fourth reversal, after which levels were adjusted by $2 \%$. Ten reversals were measured, and the balanced level was taken to be the average of the last six reversals. To obtain a final balanced level, two runs were performed, the first with electrode 7 as the reference and another electrode as the test electrode, the second with electrodes in reversed roles. Final balanced current was determined by averaging the two current differences (on a dB scale). If the two current differences were not within $10 \%$ of each other, a second pair of runs was performed and an average of the four values calculated. After every TP masker electrode had been balanced to the TP reference stimulus, the MP stimulus on electrode 7 was balanced to the TP reference on the same electrode and was then used as the reference to balance the MP stimuli on the other electrodes. The result was a set of current levels in both modes across all electrode positions that produced equal loudness when in a single-electrode stimulus of the same overall rate as the eventual dual-electrode masker. The whole procedure was then repeated to balance the stimuli at the comfortably soft level (hereafter referred to as "soft"). The procedure for loudness balancing of loud level maskers was completed during one 4-hr session and soft level maskers during a separate 4-hr session (both of which included lunch and tea breaks) in all except one participant. S4 required an additional short session to complete the balancing procedure of her loud MP masker electrodes as the consistency of her responses was uncertain. Results during her next session were checked for consistency and were within $10 \%$ of her previous results.

\section{Probe detection threshold}

Unmasked probe detection thresholds were measured using a three-interval, three-alternative forced choice (3I3AFC) adaptive procedure. One randomly selected interval of the three intervals (represented on a computer screen) contained the probe stimulus. The participant was asked to decide which of the three contained the stimulus by giving a verbal response $(1,2$, or 3$)$. $\mathrm{S} 4$, who is blind, required a slightly altered procedure. The experimenter (unaware of which interval contained the sound) tapped her hand in correspondence to the three intervals, and the verbal response $(1,2$, or 3 ) was based upon which tap on the hand contained the sound. Responses were reliable using this method. Participants were instructed to make a selection even if unsure. No feedback was provided. Probe threshold was established using a two-down one-up stepping procedure that estimated the level that gave $70.7 \%$ correct detection (Levitt, 1971). Levels were adjusted by 20\% (in $\mu \mathrm{A}$ ) until the first reversal and then by $10 \%$ until the fourth reversal, after which levels were adjusted by $2 \%$. Ten reversals were obtained in total. The average of the last six reversals was taken as threshold. Unmasked probe detection threshold was measured at the start of a session and intermittently throughout the session to check for learning or fatigue effects. An average of the thresholds (usually three or four) was calculated for later analysis.

\section{Forward-masked probe detection thresholds}

A 3I3AFC procedure was used to obtain forwardmasked probe detection thresholds. Currents on masker electrodes were fixed at balanced levels (see Sec. IIC2), while probe stimulus level was adjusted using the staircase adaptive procedure described in Sec. IIC 3. Participants were instructed that each interval would contain a sound but that they should choose the one "different" interval that contained an extra sound at the end. Levels were adjusted by $20 \%$ (in $\mu \mathrm{A}$ ) until the first reversal and then by $10 \%$ until the fourth reversal, after which levels were adjusted by $2 \%$. Ten reversals were made with threshold level calculated as the average of the last six reversals. After the thresholds were obtained for all masker-probe-distances in both modes, all the thresholds were repeated using the reverse order of mode and masker-probe distance. If repeat measurements differed from the first by $>10 \%$, a further measurement was obtained. An average of the two (or three) thresholds was taken as the final masked probe detection threshold level.

\section{Long delay masked thresholds (LDMTs)}

To evaluate residual masking, a longer masker-probe delay of $220 \mathrm{~ms}$ (offset of masker to offset of probe) was used. LDMTs at minimum and maximum masker-probe distances (masker-distances 0 and 5) were measured for both masker modes with loud and soft masker levels. The same 3I3AFC procedure as described in Sec. IIC 3 was used to measure the masked threshold of the probe stimulus. Participants were informed that stimuli would be noticeably different from the previous experiment, reflecting the increased masker-probe delay. Therefore suprathreshold training was given to each participant to ensure they were aware of the difference before the procedure continued. All participants were able to perform the task.

\section{RESULTS}

\section{A. Loudness balancing across electrodes}

Figure 3 shows balanced loud and soft levels on all masker electrodes for each participant in MP and TP modes. A $t$-test was used to compare the means of the threshold measurements for TP with the mean of those obtained using MP for each participant. Mean thresholds in all cases were significantly higher for TP than for MP (ranges of $t$ : -27.204 to $-119.429 ; p<0.001$ for all participants), consistent with other studies, i.e., higher current levels in narrower electrode configurations: BP (Kwon and van den Honert, 2006) and TP (Bierer and Faulkner, 2010). Comfortably loud levels were achieved for all participants and for all electrodes within the compliance limits of the implant (see Table II for impedance measurements). For two participants ( $\mathrm{S} 2$ and $\mathrm{S} 10)$, it was not possible to ascertain MAL in TP mode on all channels due to compliance limits. However, MALs were determined only to ensure that maximum tolerable loudness was not exceeded in the loudness-balance task, and currents at MAL were not used during the experiment. 


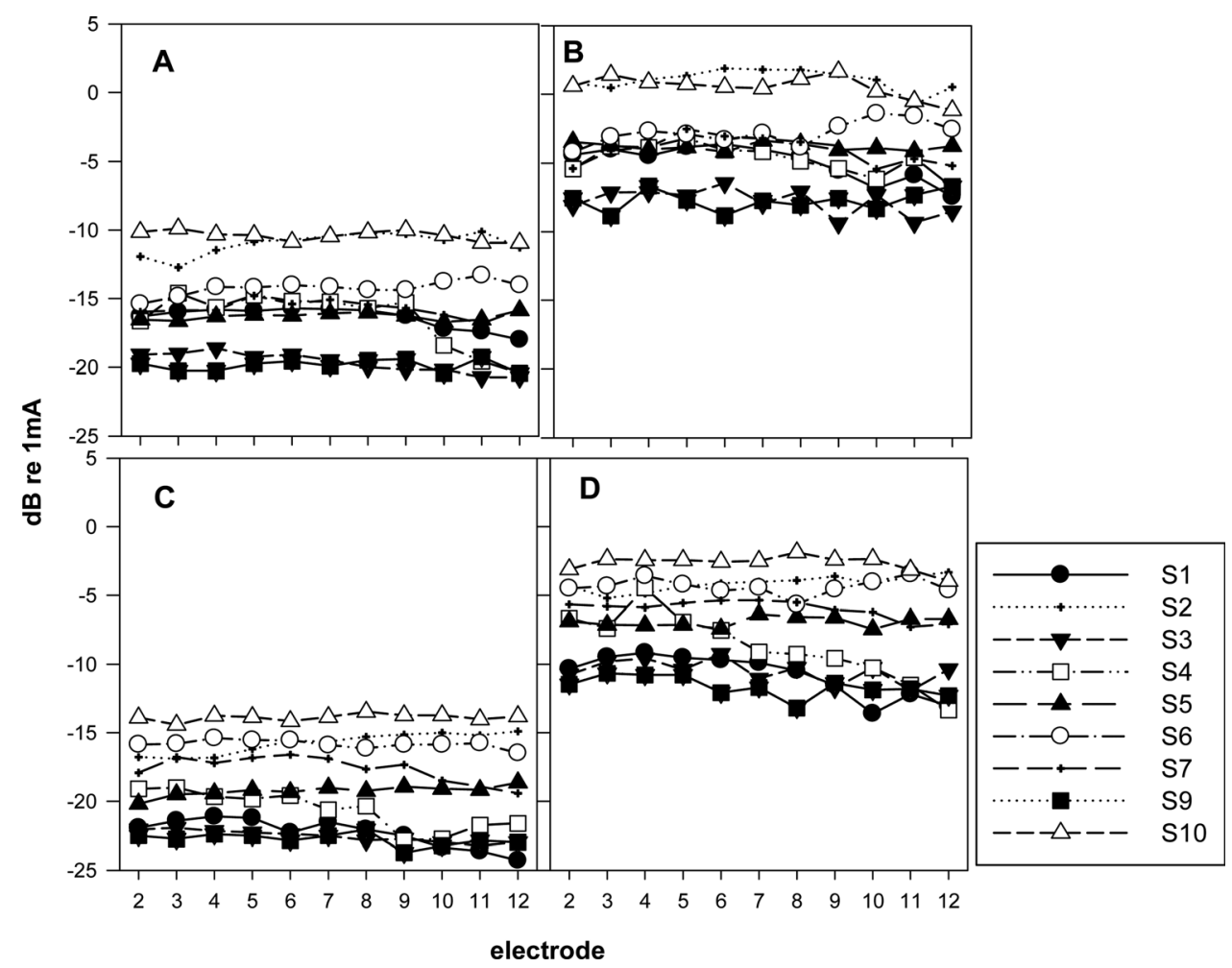

FIG. 3. Balanced levels shown in dB re $1 \mathrm{~mA}$ in both $\mathrm{MP}$ and TP modes. (A) MP loud; (B): TP loud; (C): MP soft; (D): TP soft. Note that S9 was fitted with a Helix electrode array, while the remaining participants were fitted with a HiFocus $1 \mathrm{~J}$.

\section{B. Probe thresholds}

Raw data comprising masked and not-masked probe detection thresholds for both short and long masker-probe temporal offsets are shown in Figs. 4 (loud maskers) and 5 (soft maskers). Measurements were analyzed using a logarithmic scale $(\mathrm{dB})$ as it is difficult to interpret linear current changes (subtraction in $\mu \mathrm{A}$ ) in the context of stimulus change at the neural interface (McKay, 2012). One participant (S9) fitted with the Helix electrode array, showed an atypical pattern of results with minimal masking from the dual-electrode maskers. No reasonable explanation could be provided for the lack of masking, and her results could not easily be interpreted to obtain a specificity measure, therefore her data, although shown in the figures, were excluded from the means or analyses of place specificity presented in the following text.

TABLE II. Impedance values measured on each electrode in kilohms $(\mathrm{k} \Omega)$. Impedance values were used to calculate maximum current available using the equation $I=V / R$, where $I$ is the current, $V$ is the maximum voltage of the implant (8 V), and $R$ is the impedance value in ohms.

\begin{tabular}{lllllllllllll}
\hline \hline & E2 & E3 & E4 & E5 & E6 & E7 & E8 & E9 & E10 & E11 & E12 & \\
\hline S1 & 2.7 & 3.2 & 2.9 & 3.2 & 2.9 & 2.9 & 3.2 & 2.9 & 6.9 & 7.2 & 6.4 & \\
S2 & 6.4 & 7.2 & 6.1 & 4.8 & 5.3 & 4.3 & 4.3 & 5.1 & 5.9 & 6.4 & 5.3 & \\
S3 & 5.1 & 6.4 & 5.9 & 5.3 & 6.4 & 6.7 & 6.7 & 5.6 & 6.7 & 6.9 & 5.9 & \\
S4 & 6.7 & 5.9 & 5.9 & 6.1 & 7.2 & 7.4 & 9.3 & 7.4 & 7.4 & 8.2 & 7.4 & \\
S5 & 4.5 & 4.8 & 4.0 & 4.3 & 4.3 & 5.1 & 4.8 & 4.0 & 4.0 & 4.0 & 3.7 & $\mathrm{k} \Omega$ \\
S6 & 5.9 & 5.3 & 5.6 & 5.1 & 5.6 & 6.1 & 5.6 & 7.2 & 7.4 & 8.0 & 7.4 & \\
S7 & 3.7 & 7.9 & 7.4 & 8.7 & 9.5 & 8.7 & 9.0 & 10.6 & 9.5 & 8.5 & 7.9 & \\
S9 & 4.3 & 5.0 & 4.8 & 4.3 & 4.5 & 4.8 & 4.3 & 4.5 & 5.0 & 5.0 & 4.3 & \\
S10 & 3.2 & 2.7 & 2.7 & 3.5 & 4.3 & 4.3 & 4.5 & 4.8 & 5.1 & 5.3 & 4.8 & \\
\hline \hline
\end{tabular}

\section{Spatial forward masking functions}

This section refers to masked probe detection thresholds using the short masker-probe delay $(30 \mathrm{~ms})$. The greatest degree of masking occurred when the dual-electrode masker was spatially proximal to the probe electrode and decreased with increasing spatial separation, consistent with other studies (Chatterjee and Shannon, 1998; Boex et al., 2003; Kwon and van den Honert, 2006). Threshold shifts (difference in $\mathrm{dB}$ between masked and not-masked probe thresholds) were normalized by dividing them by the threshold shift in $\mathrm{dB}$ at masker-probe distance 0 . This allowed easy comparison between participants (plotted in Figs. 6 and 7 for loud and soft maskers, respectively) and theoretically (ignoring residual masking for now) removed any effects of temporal recovery from masking from the analysis of place specificity (McKay, 2012). Some participants demonstrated a large degree of masking even at the maximum masker-probe distance. In several cases, masking remained above $60 \%$ of the maximum masking, and in one participant (S5 in MP mode and loud masker condition), masking remained above $70 \%$ of the maximum at the greatest masker-probe distance.

\section{Effect of mode and level on place specificity}

For the measurement method used in this study, place specificity (without the influence of temporal decay and ignoring residual masking) can be described by either the slope of the normalized masking function or by its width at a fixed proportion of maximum masking (McKay, 2012). Here place specificity was defined as the width of the masking function measured at $75 \%$ of the maximum masking, as this level ensured that all participants contributed to the data. It is accepted that this figure is arbitrary, and results may have 

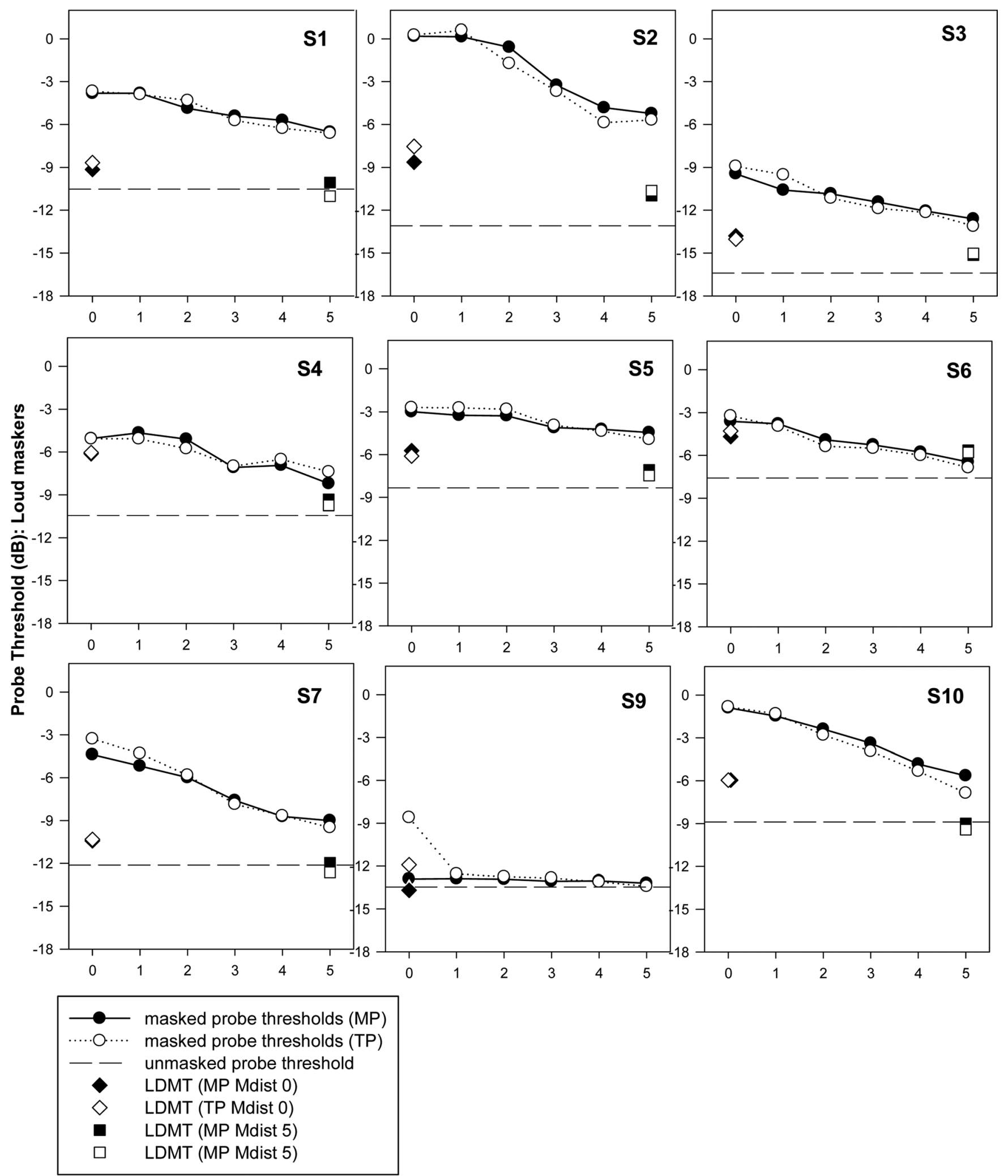

FIG. 4. Not-masked and masked probe detection thresholds for all participants using loud maskers. Not-masked probe thresholds are plotted as a continuous dashed line. Masked probe thresholds indicate the probe threshold following presentation of a MP (filled circles) or TP (open circles) dual-electrode masker at balanced "loud" levels. Also plotted are the long delay masked thresholds (LDMT), where the onset of the probe is 200 ms following the offset of the masker pulse train. A masker-probe distance of zero (Mdist 0) indicates a masker pulse train on the probe electrode (filled diamonds corresponding to MP and open to TP), and a masker-probe distance of five (Mdist 5) indicates a masker pulse train on electrodes 2 and 12 (filled squares corresponding to MP and open to TP). Note that in the Helix electrode array (S9), with a center-center electrode distance of $0.85 \mathrm{~mm}$, Mdist $1=0.85 \mathrm{~mm}$, Mdist $2=1.7 \mathrm{~mm}$, Mdist $3=2.55 \mathrm{~mm}$, Mdist $4=3.4 \mathrm{~mm}$, and Mdist $5=4.25 \mathrm{~mm}$. In the HiFocus $1 \mathrm{~J}$ electrode array (remaining participants) with a center-center electrode distance of $1.1 \mathrm{~mm}$, Mdist $1=1.1 \mathrm{~mm}$, Mdist $2=2.2 \mathrm{~mm}$, Mdist $3=3.3 \mathrm{~mm}$, Mdist $4=4.4 \mathrm{~mm}$, and Mdist $5=5.5 \mathrm{~mm}$. S9 was not included in the analyses. 
masker probe distance
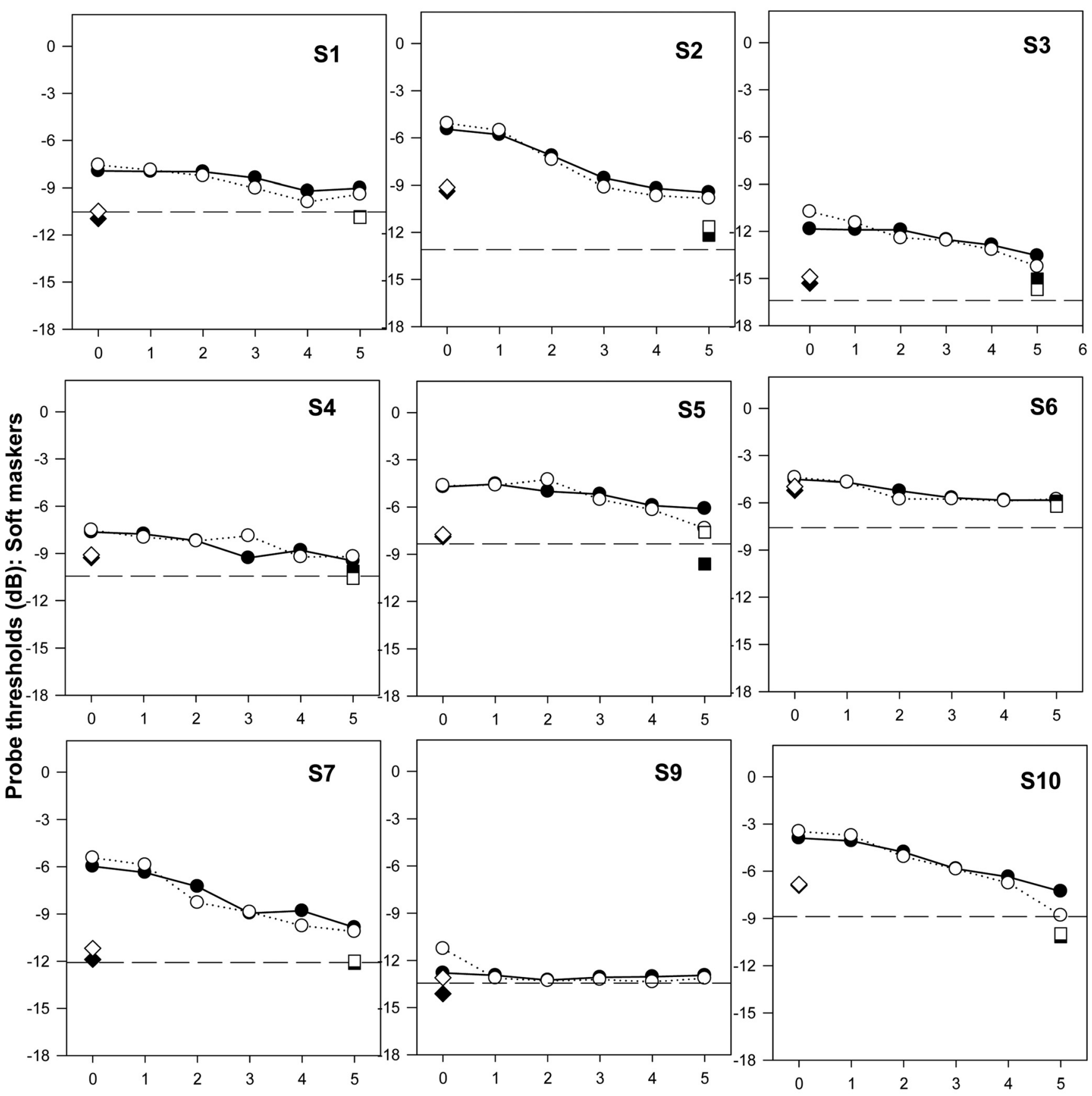

(1)

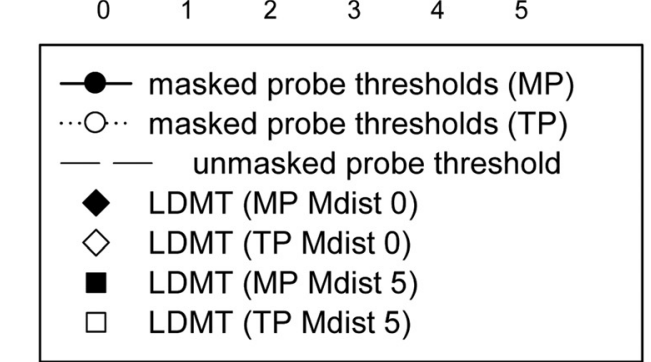

FIG. 5. Not-masked and masked probe detection thresholds for all participants using soft maskers. Not-masked probe thresholds are plotted as a continuous dashed line. Masked probe thresholds indicate the probe threshold following presentation of a MP (filled circles) or TP (open circles) masker stimulus at balanced "soft" levels. Also plotted are the long delay masked thresholds (LDMT), where the onset of the probe is $200 \mathrm{~ms}$ following the offset of the masker pulse train. A masker-probe distance of zero (Mdist0) indicates a masker pulse train on the probe electrode (filled diamonds corresponding to MP and open to TP), and a masker-probe distance of five (Mdist 5) indicates a masker pulse train on electrodes 2 and 12 (filled squares corresponding to MP and open to TP). Note that in the Helix electrode array (S9), with a center-center electrode distance of $0.85 \mathrm{~mm}$, Mdist $1=0.85 \mathrm{~mm}$, Mdist $2=1.7 \mathrm{~mm}$, Mdist $3=2.55 \mathrm{~mm}$, Mdist $4=3.4 \mathrm{~mm}$, and Mdist $5=4.25 \mathrm{~mm}$. In the HiFocus $1 \mathrm{~J}$ electrode array (remaining participants) with a center-center electrode distance of $1.1 \mathrm{~mm}$, Mdist $1=1.1 \mathrm{~mm}$, Mdist $2=2.2 \mathrm{~mm}$, Mdist $3=3.3 \mathrm{~mm}$, Mdist $4=4.4 \mathrm{~mm}$, and Mdist $5=5.5 \mathrm{~mm}$. S9 was not included in the analyses. 
masker-probe distance

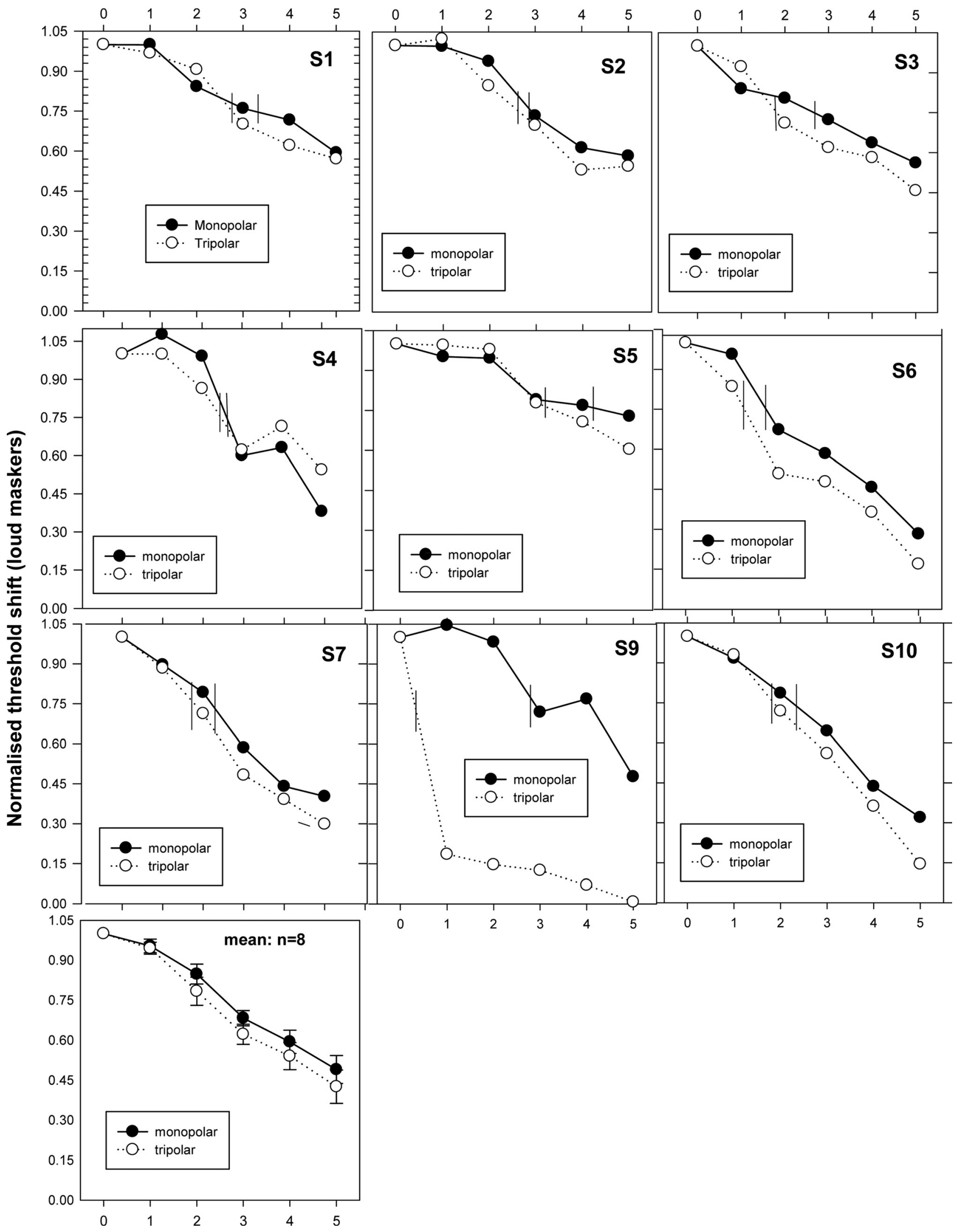

FIG. 6. Normalized threshold shifts for all participants with dual electrode masker set to "loud" level. Pairs of vertical lines show the width in masker-probe distance at $75 \%$ of the maximum threshold shift for each mode. Error bars in the mean graph denote standard errors. Note that in the Helix electrode array (S9), with a center-center electrode distance of $0.85 \mathrm{~mm}$, Mdist $1=0.85 \mathrm{~mm}$, Mdist $2=1.7 \mathrm{~mm}$, Mdist $3=2.55 \mathrm{~mm}$, Mdist $4=3.4 \mathrm{~mm}$, and Mdist $5=4.25 \mathrm{~mm}$. In the HiFocus $1 \mathrm{~J}$ electrode array (remaining participants) with a center-center electrode distance of $1.1 \mathrm{~mm}$, Mdist $1=1.1 \mathrm{~mm}$, Mdist $2=2.2 \mathrm{~mm}$, Mdist $3=3.3 \mathrm{~mm}$, Mdist $4=4.4 \mathrm{~mm}$, and Mdist $5=5.5 \mathrm{~mm}$. S9 was not included in the analyses or mean graph. 
Masker Probe Distance
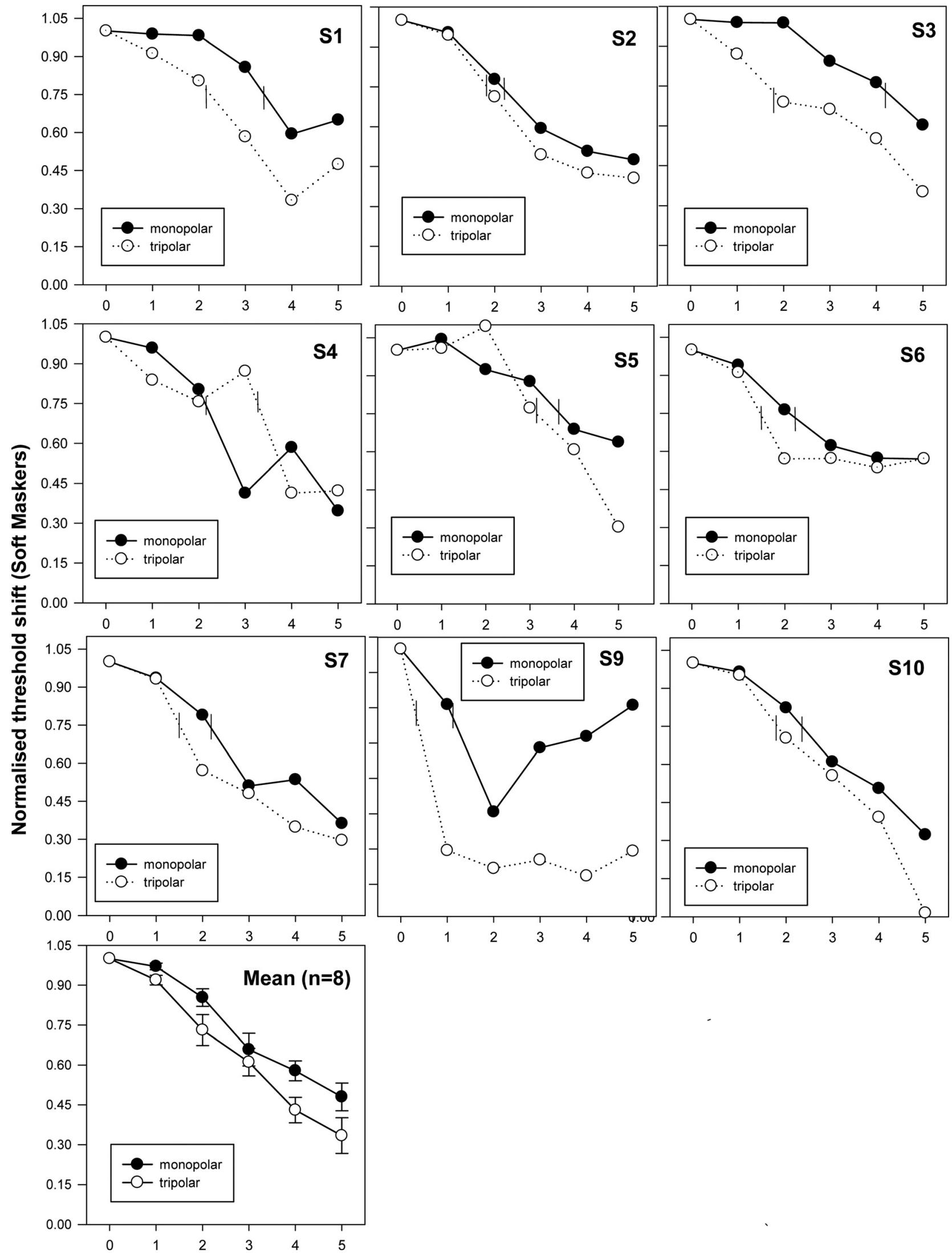

FIG. 7. Normalized threshold shifts for all participants with dual electrode masker set to "soft" level. Pairs of vertical lines show the width in masker-probe distance at $75 \%$ of the maximum threshold shift for each mode. Error bars in the mean graph denote standard errors. Note that in the Helix electrode array (S9), with a center-center electrode distance of $0.85 \mathrm{~mm}$, Mdist $1=0.85 \mathrm{~mm}$, Mdist $2=1.7 \mathrm{~mm}$, Mdist $3=2.55 \mathrm{~mm}$, Mdist $4=3.4 \mathrm{~mm}$, and Mdist $5=4.25 \mathrm{~mm}$. In the HiFocus $1 \mathrm{~J}$ electrode array (remaining participants) with a center-center electrode distance of $1.1 \mathrm{~mm}$, Mdist $1=1.1 \mathrm{~mm}$, Mdist $2=2.2 \mathrm{~mm}$, Mdist $3=3.3 \mathrm{~mm}$, Mdist $4=4.4 \mathrm{~mm}$, and Mdist $5=5.5 \mathrm{~mm}$. S9 was not included in the analyses or mean graph. 
differed if another measure of place specificity was used. Results are plotted in Fig. 8. The width of the masking functions at $75 \%$ of the maximum masking ranged from 1.41 to 3.38 electrodes $(1.55-3.72 \mathrm{~mm})$ for $\mathrm{TP}$ and 1.92 to 4.47 $(2.11-4.92 \mathrm{~mm}$ ) for MP (loud maskers) and from 1.47 to 3.25 electrodes $(1.62-3.58 \mathrm{~mm}$ ) for TP and 2.09 to 4.17 electrodes (2.3-4.59 mm) for MP (soft maskers).

A two-way, repeated-measures analysis of variance (ANOVA) with factors of mode (TP or MP) and masker level (loud or soft) was conducted. A significant effect of mode was found $[\mathrm{F}(1,7)=7.402 ; p=0.030]$, indicating better place specificity of TP compared to MP mode. There was no effect of masker level on place specificity and no interaction between mode and masker level $(\mathrm{F}=0.320 ; p=0.589$ and $\mathrm{F}=0.234 ; p=0.643$, respectively).

\section{E. Residual masking}

Most participants demonstrated a degree of residual masking (see Figs. 4 and 5). Means, standard deviations and ranges of residual masking are displayed in Table III.

To investigate whether loud maskers produced more residual masking than soft maskers at masker-probe distance 0, a one-way repeated-measures ANOVA was performed, comparing residual masking (averaged across modes) for loud and soft maskers $(n=8)$. Loud maskers (mean of $2.96 \mathrm{~dB}$ ) produced a significantly higher amount of residual masking than soft maskers (mean of $1.48 \mathrm{~dB})(\mathrm{F}=28.503$; $p=0.001)$.

To determine the influence of residual masking on the normalized forward masking functions (Figs. 6 and 7) and hence on our measures of specificity (Fig. 8), the amount of residual masking was converted to a proportion of the maximum masking (normalized) before further analysis. A withinsubjects three-way repeated measures ANOVA with factors of mode, level, and masker-probe distance was conducted. This showed no effect of mode $[\mathrm{F}(1,7)=0.91 ; p=0.772]$, an effect of masker-probe distance $[\mathrm{F}(1,7)=24.16 ; p=0.002]$

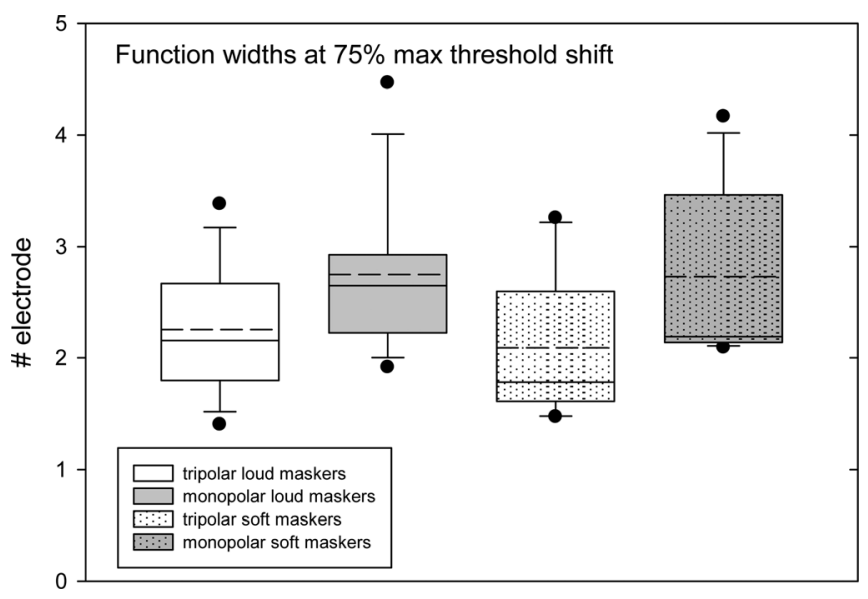

FIG. 8. Box plot illustrating spatial masking function widths for loud and soft TP and MP maskers at $75 \%$ of the maximum threshold shift. The boxes demonstrate the 25th, 50th, and 75th percentiles, with the mean value shown as a dashed line. Whiskers indicate the 5 th and 95 th percentile values. Outliers are shown as individual points. Electrode numbers relate to the HiFocus $1 \mathrm{~J}$ electrode array with a center-center electrode distance of $1.1 \mathrm{~mm},(1=1.1 \mathrm{~mm}$, $2=2.2 \mathrm{~mm}, 3=3.3 \mathrm{~mm}, 4=4.4 \mathrm{~mm}$, and $5=5.5 \mathrm{~mm}$ ).
TABLE III. Mean residual masking across subjects as a function of mode (TP or MP), masker level (loud or soft) and masker-probe distance ( 0 or 5 ). Standard deviations (SD) and range of residual masking in decibels are also shown.

\begin{tabular}{llccccc}
\hline \hline $\begin{array}{l}\text { Mode of } \\
\text { stimulation }\end{array}$ & $\begin{array}{c}\text { Masker } \\
\text { level }\end{array}$ & $\begin{array}{c}\text { Masker-probe } \\
\text { distance }\end{array}$ & $\begin{array}{c}\text { Mean } \\
(\mathrm{dB})\end{array}$ & $\begin{array}{c}\text { SD } \\
(\mathrm{dB})\end{array}$ & $\begin{array}{c}\text { Minimum } \\
(\mathrm{dB})\end{array}$ & $\begin{array}{c}\text { Maximum } \\
(\mathrm{dB})\end{array}$ \\
\hline $\mathrm{MP}$ & Loud & 0 & 2.86 & 1.09 & 1.38 & 4.46 \\
$\mathrm{MP}$ & Loud & 5 & 1.03 & 0.8 & -0.1 & 2.13 \\
$\mathrm{TP}$ & Loud & 0 & 3.06 & 1.32 & 1.8 & 5.56 \\
$\mathrm{TP}$ & Loud & 5 & 0.7 & 1.14 & -0.55 & 2.46 \\
$\mathrm{MP}$ & Soft & 0 & 1.32 & 1.33 & -0.43 & 3.71 \\
$\mathrm{MP}$ & Soft & 5 & 0.18 & 1.12 & 1.28 & 1.7 \\
$\mathrm{TP}$ & Soft & 0 & 1.63 & 1.24 & 0.04 & 3.96 \\
$\mathrm{TP}$ & Soft & 5 & 0.35 & 0.88 & 1.09 & 1.46 \\
\hline \hline
\end{tabular}

and an effect of level that was approaching significance $[\mathrm{F}(1,7)=4.826 ; p=0.064]$. There were no significant interactions. In summary, the amount of normalized residual masking did not vary significantly with mode or masker level but did vary with masker-probe distance. Therefore although the slopes of the spatial masking functions would have differed if residual masking was taken into account (i.e., if the threshold shift was calculated relative to the LDMT rather than the not-masked probe threshold), the outcomes of the previous analyses of the effects of mode and masker level on place specificity (Sec. III D) are unlikely to have differed.

Residual masking was not likely to influence the result of analyses of specificity within the same subject for different modes. However, it is likely to influence the absolute value of the specificity measure in different ways for different subjects. Figure 9 illustrates two cases in which residual masking affected width measurements to a differing degree. The plotted data are normalized threshold shifts relative to both the not-masked probe threshold (circles), as in Figs. 6 and 7 , and relative to the LDMTs (triangles). Because LDMTs were measured only at masker probe distances of 0 and 5, linearly interpolated values were used to estimate LDMTs for the remaining masker-probe distances. For S2, the effect of residual masking on the width measurement was small when using both loud [Fig. 9(A)] and soft [Fig. 9(C)] masker levels as was the effect on the slope of the masking function. Conversely, for S10, the effect of residual masking on both the width and slope of the masking function is considerable when using loud [Fig. 9(B)] and particularly soft [Fig. 9(D)] masker levels. It is possible that linear interpolation (of the amount of residual masking at masker-probe distances other than 0 or 5) exaggerated or diminished the effect of true residual masking, and therefore the data for these two participants were plotted using this method only for illustration purposes. It would be necessary to measure LDMTs at all masker-probe distances to fully take into account residual masking. Nevertheless, the results from these two participants illustrate that residual masking may be highly variable between individuals and as such cannot be disregarded in forward masking experiments comparing place specificity across subjects. If residual masking is a cognitive effect, it should be first removed from the threshold shift before analyzing the forward masking functions, i.e., 


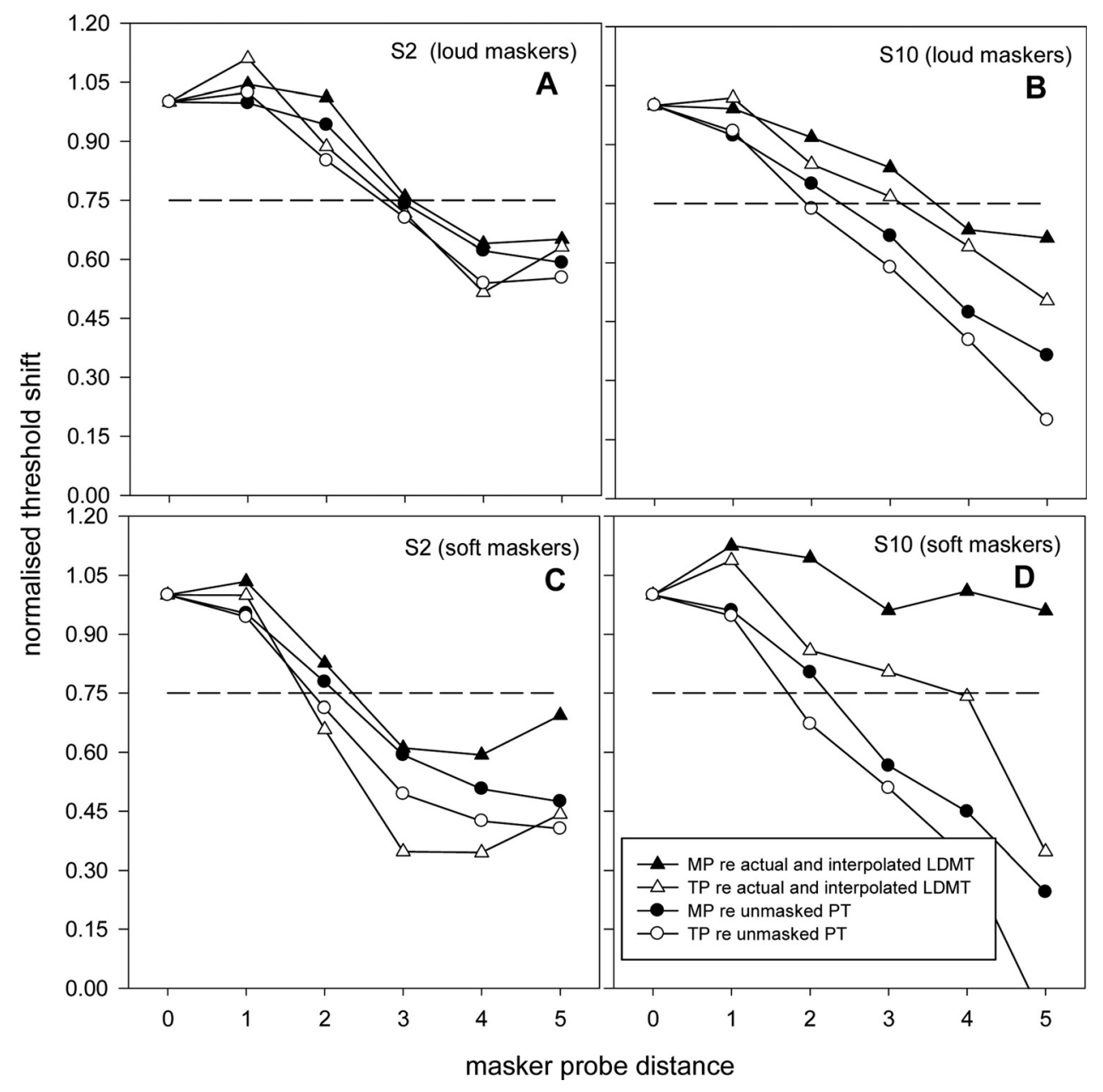

FIG. 9. Examples of spatial forward masking functions from two participants to illustrate the effect of taking residual masking into account. In (A) and (C) (S2 with loud and soft maskers, respectively), the difference in width measurement as measured by the $75 \%$ threshold shift point (dashed line) is negligible. In (B) and (D), however, (S10 with loud and soft maskers, respectively), the threshold shift at $75 \%$ of the maximum masking is considerably different depending upon whether it is relative to the not-masked probe threshold or the LDMT. the threshold shift should be referenced to the LDMT, not the not-masked threshold, as has been typically the case in previously published forward masking experiments.

To summarize, most participants demonstrated measurable residual masking at $220 \mathrm{~ms}$ masker-probe delay. When normalized by converting to a proportion of maximum threshold shift, the amount of residual masking was significantly greater at a masker-probe distance of 0 than a maskerprobe distance of 5. There was no effect of masker level (that is, the amount of residual masking tended to be a fixed proportion of the maximum masking at each masker level), and crucially the degree of normalized residual masking did not differ with mode. Therefore the main results of this study (differences in place specificity observed between modes and lack of effect of level on place specificity) would likely not have differed if residual masking were taken into account by measuring it at all masker-probe distances.

\section{DISCUSSION}

The present study showed that, in a group of Advanced Bionics cochlear implant users, using a dual-electrode masker forward masking paradigm with component masker electrodes balanced for loudness, a TP stimulus $(\sigma: 0.75)$ produced more place-specific neural activity than a MP stimulus. However, as in previous studies (Bierer and Faulkner, 2010; Zhu et al., 2012; Landsberger et al., 2012), the effect of mode was small and varied among subjects. More research is needed to ascertain whether this effect may be clinically significant in everyday speech processing.

Use of a single-electrode masker, as in previous studies, can significantly sharpen the function via detection of the probe excitation at sites distal from the probe electrode and thus place specificity can be overestimated using that technique. A limitation of using a dual-electrode masker with a fixed probe position, as used here, is that it does not allow identification of asymmetries in the masking on either side of the probe electrode. If the method was expanded so that probe thresholds were measured for a series of probe positions between the two masker electrodes (instead of a single central probe position), this limitation would be overcome. The limitation of off-site listening by using the dualelectrode masker, although theoretically preventing off-site listening from artificially favoring MP mode, did not lead to more consistent benefits of TP across subjects than seen in previous studies. Poor specificity may occur if the neural survival in the cochlea is relatively sparse, leading to neural activity near the probe electrode saturating as current is increased and necessitating further increases of current (thus wider activation patterns) to achieve the required loudness.

Bierer and Faulkner (2010) found that channels with lower threshold levels were associated with better selectivity than channels with higher thresholds, suggesting that good electrode-neuron interface (reflecting nerve survival or 
electrode placement) may be fundamental in delivering focused stimulation. Thus measuring thresholds with a narrow electrode configuration could be a useful way to identify "problem" electrodes with poor neural interface (Bierer, 2010). In the present data, a Pearson correlation revealed no significant relationship across subjects between TP notmasked probe thresholds and place specificity assessed using either loud or soft masker levels $(\mathrm{r}=0.0293 ; p=0.945$, using loud maskers; $\mathrm{r}=0.193 ; p=0.646$ using soft maskers), showing that the within- and across-subject effects may be influenced by different factors. It is interesting to speculate whether the relationship found by Bierer and Faulkner (2010) might have been influenced by the specificity measure used (the slope in $\mathrm{dB} / \mathrm{mm}$ of the absolute masker levels that just masked the fixed probe). The lowest TP probe threshold is likely to be at the same electrode position as the lowest masker threshold. Thus the masker thresholds may have sloped upward from the probe position with the lowest threshold, artificially adding to the slope in $\mathrm{dB} / \mathrm{mm}$ of the masking function at that probe position. Whether this potential influence would change the outcome of the experiment cannot be known unless the data were re-analyzed to express the masker levels as level re masker threshold or \%DR.

The data showed no mean effect of masker level on place specificity in either mode. This finding is in agreement with Chatterjee et al. (2006) and Zhu et al. (2012), who found that the shape of the forward masking pattern did not change significantly with masker level. However, inspection of Figs. 6 and 7 reveals that increases and decreases in place specificity occur in different subjects and different modes, and this variability between subjects is also apparent in other published data (e.g., Nelson et al., 2008). The variability is likely to be due to variability in neural survival patterns as the specificity of the current field does not theoretically change with current amplitude.

Spatial forward masking functions in cochlear implant listeners typically show non-uniform shapes and even nonmonotonicities, possibly the result of patchy neural survival (Bierer, 2009, 2010; Bierer et al., 2010). A difficulty, not easily addressed with any such non-smooth spatial masking function, is to know which place specificity measure is most related to perceptual resolution of place or frequency. The measure chosen in this study was the width of the masking function at $75 \%$ of the maximum masking. It is not clear whether results may have differed if a different measure of specificity was used (a width measurement at a different percentage, or a slope measurement at a certain point of the function). More research is needed to examine how different psychophysical measures of specificity relate to perceptual resolution in electrical hearing.

In the present study, forward masking was used not only to assess place specificity but also to evaluate the effect of residual masking on the measures of place specificity. A significantly greater amount of residual masking was found when the masker and probe stimuli were on the same electrode (Mdist 0) than when they were more distal (Mdist 5). Theoretically, the threshold shift in the forward masking functions should be determined relative to the residual masking threshold, as estimated in Fig. 9 for two participants rather than the unmasked threshold. A linear interpolation was used to estimate residual masking for masker-probe distances between 1 and 4 in that figure; however, it is unclear what the true effect of masker-probe distance on residual masking would be for the in-between range of distances. It is plausible that, as the masker and probe become more perceptually dissimilar, less cognitive effort would be needed to listen for the probe stimulus, resulting in smaller degree of residual making. Further research is needed to investigate the effect of masker-probe distance on residual masking. However, it is clear that the effect of residual masking on the shape of the masking function will be subject dependent and therefore important to consider in experiments that compare specificity between subjects. Evaluation of the degree of normalized residual masking revealed no average effect of mode, thus improvements in within-subject place specificity observed in TP compared to MP mode in this study are unlikely to be largely influenced by unconsidered contributions of residual masking in each mode.

The mechanism underlying residual masking is poorly understood. It is hypothesized to be the byproduct of a cognitive or attentional process (i.e., the effect of shifting attention from a loud masker stimulus to a threshold probe stimulus). McKay (2012) discussed the possible effects of residual masking on place specificity measures and suggested that if the degree of residual masking differed among subjects, it could not only influence the accuracy of the specificity measure but also, importantly, any correlation of specificity with other subject characteristics such as speech perception. If residual masking was a cognitive effect, and if cognitive effort was equal for each probe detection task, it would be constant for different masker-probe distances (McKay, 2012). The present findings, showing a different degree of residual masking at two different masker-probe distances (Figs. 4 and 5), may still support the hypothesis that residual masking is due to cognitive factors if less cognitive effort is required to discriminate a probe from a perceptually distinct masker stimulus (Mdist 5) than from a perceptually similar masker stimulus (Mdist 0). Moore and Glasberg $(1981,1982)$ suggested that if the masker is the same frequency as the probe, the probe may sound like an extension in the duration of the masker: a task of duration discrimination rather than probe detection. Loud masker levels produced significantly more residual masking than soft masker levels, consistent with Nelson and Donaldson (2002), who demonstrated a significant increase in residual masking over three masker levels (see McKay, 2012 for calculation) and provided support for the hypothesis that the loudness of the masker may contribute to the degree of cognitive effort required to listen for the threshold probe.

The mean amount of residual masking when masker and probe were on the same electrode (Mdist 0) was compared with two published recovery function experiments. Nelson and Donaldson (2002) and Dingemanse et al. (2006) measured recovery from forward masking using various maskerprobe offsets and represented residual masking as an additive fitting constant in the model of masking decay. These constants (first converted to $\mathrm{dB}$ from $\mu \mathrm{A}$ ) were compared with the present amounts of residual masking at a comparable 
masker loudness [loud in Nelson and Donaldson (2002) and "midrange amplitude" in Dingemanse et al. (2006)]. The present results showed a greater amount of residual masking (with standard deviation): 2.9 (1.18) $\mathrm{dB}$, compared with 1.04 (0.44) dB (Dingemanse et al., 2006) and 1.9 (0.9) dB (Nelson and Donaldson, 2002). The larger residual masking found in the present experiment may be associated with the fact that the measurement was taken at a single masker-probe offset, whereas the two studies discussed in the preceding text derived the residual masking as a fitted asymptotic value of the masking recovery function. It is possible that full decay to the residual constant was not achieved in the current experiment by $220 \mathrm{~ms}$. Results of Nelson and Donaldson (2002) and Dingemanse et al. (2006) suggested that by $220 \mathrm{~ms}$ offset, masking had decayed to the residual constant in most but not all cases. Perhaps to completely eliminate this possibility, a longer time delay for the LDMTs may have been more appropriate in this experiment. The greater residual masking in the present experiment may also be the result of using higher rates and hence lower currents than the other studies, where the loudness versus current slope is shallower (McKay et al., 2003). In that case, a larger change in current would be needed to produce an equal change in loudness. The present experiment adopted a high rate of stimulation for the masker and the probe stimuli to ensure current levels remained low: an important consideration in focused stimulation where there may be associated compliance issues.

\section{CONCLUSIONS}

TP mode was found to be more place-specific than MP mode, but the effect was not uniformly large across participants. A dual-electrode masker stimulus controlled the offsite listening cues but did not increase the consistency of the mode difference across subjects compared to previous studies, suggesting that the failure of the TP mode to produce significantly greater specificity in all subjects may be due to poor neural survival patterns.

The influence of residual masking on spatial forward masking functions was demonstrated, and this factor is important to consider when comparing spatial specificity across subjects.

\section{ACKNOWLEDGMENTS}

This study was financially supported by a CASE studentship provided by the UK Economic and Social Research Council and by Advanced Bionics Corporation and is documented in a Ph.D. thesis written by the first author. We thank Advanced Bionics Corporation for their ongoing support and advice as well as for the equipment and software needed to perform the experiment. We thank the Midlands Hearing Implant Programme for providing the details provided in Table I. We would like to thank Chris Plack for his comments on an earlier manuscript. We are particularly grateful to the participants for their continued participation in our research.

Bench, J., Kowal, A., and Bamford, J. (1979). "The BKB (Bamford-KowalBench) sentence lists for partially hearing children,” Br. J. Audiol 13(3), $108-112$.
Bierer, J. (2007). "Threshold and channel interaction in cochlear implant users: Evaluation of the tripolar electrode configuration," J. Acoust. Soc. Am. 121, 1642-1653.

Bierer, J. A. (2009). "Identifying channels with poor electrode-neuron interfaces: Using the partial-tripolar configuration," in Conference on Implantable Auditory Prostheses, Lake Tahoe, CA.

Bierer, J. A. (2010). "Probing the electrode-neuron interface with focused cochlear implant stimulation," Trends. Amplif. 14, 84-95.

Bierer, J. A., Bierer, S. M., and Middlebrooks, J. C. (2010). "Partial tripolar cochlear implant stimulation: Spread of excitation and forward masking in the inferior colliculus," Hear. Res. 270, 134-142.

Bierer, J. A., and Faulkner, K. F. (2010). "Identifying cochlear implant channels with poor electrode-neuron interface: Partial tripolar, singlechannel thresholds and psychophysical tuning curves," Ear Hear. 31(2), $1-12$.

Bierer, J. A., and Middlebrooks, J. C. (2002). "Auditory cortical images of cochlear-implant stimuli: Dependence on electrode configuration," J. Neurophysiol. 87, 478-492.

Boex, C., Kos, M., and Pelizzone, M. (2003). "Forward masking in different cochlear implant systems," J. Acoust. Soc. Am. 114, 2058-2065.

Chatterjee, M., Galvin, J. J., III, Fu, Q. J., and Shannon, R. V. (2006). "Effects of stimulation mode, level and location on forward-masked excitation patterns in cochlear implant patients," J. Assoc. Res. Otolaryngol. 7, 15-25.

Chatterjee, M., and Shannon, R. (1998). "Forward masked excitation patterns in multielectrode electrical stimulation," J. Acoust. Soc. Am. 103, 2565-2572.

Cohen, L. T., Busby, P. A., and Clark, G. M. (1996). "Cochlear implant place psychophysics. II. Comparison of forward masking and pitch estimation data," Audiol. Neurootol. 1, 278-292.

Cohen, L. T., Richardson, L. M., Saunders, E., and Cowan, R. S. (2003). "Spatial spread of neural excitation in cochlear implant recipients: Comparison of improved ECAP method and psychophysical forward masking," Hear. Res. 179, 72-87.

de Balthasar, C., Boex, C., Cosendai, G., Valentini, G., Sigrist, A., and Pelizzone, M. (2003). "Channel interactions with high-rate biphasic electrical stimulation in cochlear implant subjects," Hear Res. 182, 77-87.

Dingemanse, J. G., Frijns, J. H., and Briaire, J. J. (2006). "Psychophysical assessment of spatial spread of excitation in electrical hearing with single and dual electrode contact maskers," Ear Hear. 27, 645-657.

Hughes, M. L., and Stille, L. J. (2008). "Psychophysical versus physiological spatial forward masking and the relation to speech perception in cochlear implants," Ear Hear. 29, 435-452.

Jolly, C. N., Spelman, F. A., and Clopton, B. M. (1996). "Quadrupolar stimulation for cochlear prostheses: Modeling and experimental data," IEEE Trans. Biomed. Eng. 43, 857-865.

Kral, A., Hartmann, R., Mortazavi, D., and Klinke, R. (1998). "Spatial resolution of cochlear implants: The electrical field and excitation of auditory afferents," Hear. Res. 121, 11-28.

Kwon, B. J., and van den Honert, C. (2006). "Effect of electrode configuration on psychophysical forward masking in cochlear implant listeners," J. Acoust. Soc. Am. 119, 2994-3002.

Landsberger, D. M., Padilla, M., and Srinivasan, A. G. (2012). "Reducing current spread using current focusing in cochlear implant users," Hear. Res. 284, 16-24.

Levitt, H. (1971). "Transformed up-down methods in psychoacoustics," J. Acoust. Soc. Am. 49(2), 467-477.

Lim, H. H., Tong, Y. C., and Clark, G. M. (1989). "Forward masking patterns produced by intracochlear electrical stimulation of one and two electrode pairs in the human cochlea," J. Acoust. Soc. Am. 86, 971-980.

Macherey, O., van Wieringen, A., Carlyon, R. P., Dhooge, I., and Wouters, J. (2010). "Forward-masking patterns produced by symmetric and asymmetric pulse shapes in electric hearing," J. Acoust. Soc. Am. 127, 326-338.

McKay, C. M. (2012). "Forward masking and place specificity of neural excitation in cochlear implants: A review of methods and interpretation," J. Acoust. Soc. Am. 131, 2209-2224.

McKay, C. M., Henshall, K. R., Farrell, R. J., and McDermott, H. J. (2003). "A practical method of predicting the loudness of complex electrical stimuli,” J. Acoust. Soc. Am. 113, 2054-2063.

Middlebrooks, J. C. (2004). "Effects of cochlear-implant pulse rate and inter-channel timing on channel interactions and thresholds," J. Acoust. Soc. Am. 116, 452-468.

Moore, B. C., and Glasberg, B. R. (1981). "Auditory filter shapes derived in simultaneous and forward masking," J. Acoust. Soc. Am. 70, 1003-1014. 
Moore, B. C., and Glasberg, B. R. (1982). "Contralateral and ipsilateral cueing in forward masking,” J. Acoust. Soc. Am. 71, 942-945.

Nelson, D. A., and Donaldson, G. S. (2002). "Psychophysical recovery from pulse-train forward masking in electric hearing," J. Acoust. Soc. Am. 112, 2932-2947.

Nelson, D. A., Donaldson, G. S., and Kreft, H. (2008). "Forward-masked spatial tuning curves in cochlear implant users," J. Acoust. Soc. Am. 123, $1522-1543$.

Oxenham, A. J., and Moore, B. C. (1995). "Additivity of masking in normally hearing and hearing-impaired subjects," J. Acoust. Soc. Am. 98, 1921-1934.
Seaton, W. H. (1979). "Level dependence of critical bandwidth: Notchednoise masking paradigm,” J. Aud. Res. 19, 267-276.

Stickney, G. S., Loizou, P. C., Mishra, L. N., Assmann, P. F., Shannon, R. V., and Opie, J. M. (2006). "Effects of electrode design and configuration on channel interactions," Hear. Res. 211, 33-45.

Throckmorton, C. S., and Collins, L. M. (1999). "Investigation of the effects of temporal and spatial interactions on speech-recognition skills in cochlear-implant subjects," J. Acoust. Soc. Am. 105, 861-873.

Zhu, Z., Tang, Q., Zeng, F. G., Guan, T., Ye, D. (2012). "Cochlear-implant spatial selectivity with monopolar, bipolar and tripolar stimulation," Hear. Res. 283, 45-58. 